\title{
What is the evidence for mirtazapine in treating cancer-related symptomatology? A systematic review
}

\author{
Guillaume Economos $^{1,2}\left(\right.$ D $~ \cdot$ Natasha Lovell $^{1}\left(\right.$ C) $\cdot$ Anna Johnston ${ }^{1} \cdot$ Irene J. Higginson ${ }^{1}(\mathbb{C}$
}

Received: 17 July 2019 / Accepted: 5 December 2019 / Published online: 19 December 2019

(C) The Author(s) 2019

\begin{abstract}
Purpose Cancer patients often experience multiple distressing symptoms which are challenging to manage. It would therefore be helpful to find a treatment that alleviates more than one symptom, to avoid polypharmacy: mirtazapine has been used in several studies for this purpose. The objective of this study was to assess the effectiveness and safety of mirtazapine in alleviating one or more frequently encountered cancer-related symptoms.

Methods Systematic review of clinical trials in English or French. Eight databases were searched. Included studies assessed the effectiveness of mirtazapine in alleviating one or more frequently encountered cancer-related symptoms. Comparator and validated assessment tools were required. Studies were independently appraised by two investigators before data synthesis.

Results The search yielded 1898 references, from which we identified 12 relevant articles evaluating highly heterogeneous outcomes. These were two randomised-controlled (RCTs), three non-randomised controlled, and seven non-randomised noncontrolled trials. In total, 392 participants were included and 185 were in RCTs. No study assessed the effectiveness of mirtazapine in alleviating symptoms at the same time, but some considered more than one symptom. Overall, the data was of poor quality, limited by small sample size and bias. However, mirtazapine showed effectiveness in treating depression, anxiety, sleep disorders, emesis and neuropathic pain. Across all studies, mirtazapine is safe to use, with drowsiness and dizziness the most common side-effects.

Conclusion Study design and small sample sizes limit the ability to interpret results. Trials to assess the impact of mirtazapine or other medicines in alleviating multiple symptoms would be valuable.
\end{abstract}

Keywords Mirtazapine $\cdot$ Neoplasms $\cdot$ Palliative care $\cdot$ Supportive care in cancer $\cdot$ Polysymptomatology

Electronic supplementary material The online version of this article (https://doi.org/10.1007/s00520-019-05229-7) contains supplementary material, which is available to authorized users.

Guillaume Economos

economos.guillaume@gmail.com

Natasha Lovell

Natasha.lovell@kcl.ac.uk

Anna Johnston

Anna.johnston@kcl.ac.uk

Irene J. Higginson

Irene.higginson@kcl.ac.uk

1 Cicely Saunders Institut, Departement Palliative Care Policy and Rehabilitation, King's College London, 10 Cutcombe Rd, Brixton, London SE5 9PJ, UK

2 Hospices Civils de Lyon, Centre Hospitalier Lyon Sud, Palliative Care, 165 chemin du Grand Revoyet, 69310 Pierre-Bénite, France

\section{Introduction}

Cancer patients often experience multiple distressing symptoms simultaneously [1]. The experience of multiple symptoms at the same time is referred to as polysymptomatology and requires multiple medications to mitigate their effect [2]. The most burdensome includes fatigue, pain, lack of energy, weakness and loss of appetite, affecting more than half of patients with advanced cancer $[1,3]$. These symptoms are a challenge to assess and treat, and very few drugs are licenced for this purpose $[4,5]$. In this frail and multimorbid population, polymedication increases the risk of drug interactions and side effects $[6,7]$. One approach to tackle this is identifying a single medication which can effectively treat multiple symptoms.

Mirtazapine [8], a noradrenergic and specific serotonergic antidepressant, has proved effective in the treatment of depression in the cancer population [9]. It has also been 
evaluated in several studies to alleviate other cancerrelated symptoms. This pre-synaptic $\alpha 2$ adrenoreceptor antagonist increases the central noradrenergic and serotoninergic neurotransmission. Whilst the cause of its effectiveness as an antidepressant remains unclear, it is hypothesised to be due to a blockage of pre-synaptic $\alpha 2$ receptors leading to the release of norepinephrine, and a better availability of neurotransmitters in the synapse. It also antagonizes $\alpha 2$ heteroreceptors leading to an increment of serotonin release. Besides these central noradrenergic and serotoninergic effects, mirtazapine has an affinity to the anti-H1 receptor and is an 5-HT3 antagonist [10], which could be relevant in treating sleep disorders, appetite and breathlessness [11]. With this pharmacological profile, mirtazapine may be effective for the treatment of multiple symptoms, particularly those associated with cancer $[5,8,12]$.

Mirtazapine is reported to be a safe antidepressant drug in the cancer population. It is almost completely metabolized by the liver and has a low-drug interaction risk, thus, allowing its use in advanced renal failure $[10,13]$. However, some authors report drug-related symptoms such as dry-mouth, sedation, increased appetite and weight gain [14]. Sedation, increased appetite and weight gain are specific to mirtazapine, and could be useful in the cancer population who commonly experience poor sleep and a lack of appetite.

The effectiveness and safety of mirtazapine in alleviating multiple symptoms in cancer populations remain unclear. This review aims to address this question.

\section{Material and methods}

We performed a systematic review of the literature using eight different databases to identify studies relevant to our research question.

The full protocol is available in supplementary material 1 .

\section{Data sources}

To identify relevant studies, we searched on Medline, Scopus, Web of Science, Central and EMBASE. We searched for grey literature on Clinical Trials, the WHO ICTRP and OpenGrey. Investigators were contacted by email to request any unpublished study details identified using the clinical trial databases. Additional records were identified using related articles and references as well as by open searches. The inclusion time frame covered all databases until the 15th of January 2019.

Research algorithms were designed to fit with each database to improve the sensitivity of the search (supplementary material 2). The titles and abstracts (if available) of yielded records were screened for inclusion and exclusion criteria to evaluate their eligibility. Full text articles were then read and non-relevant articles excluded.

\section{Article selection}

We included only primary literature: randomized controlled trials (RCTs), cohort studies, case-controlled and nonrandomised experimental studies, reporting original studies written in English or French languages. Experimental studies were required to use a control.

Included studies concerned patients diagnosed with cancer, excluding cancer survivors, with one or more of the following symptoms: depression, anxiety, sleep disorders, nausea, anorexia, weight loss, breathlessness, pain, constipation, fatigue and drowsiness. These symptoms were chosen based on the fact that they are the most frequently encountered symptoms in cancer [3] and could potentially be addressed using mirtazapine given its pharmacological profile [8]. The primary outcome of studies was improvement of one of the listed symptoms.

\section{Data extraction}

Data was extracted independently by two authors (GE, NL) regarding the effectiveness of mirtazapine (primary or secondary outcome) and the safety of its use. Authors extracted data on the year of publication and country of the study, number of participants, doses and modes of administration of mirtazapine and the comparator, follow-up completion rate, assessed symptoms and the tools used for assessment, results of the analysis, reported toxicity, adverse events and reasons for withdrawals.

\section{Data synthesis}

Two authors (GE, NL) independently assessed the risk of bias and quality of studies using Cochrane Collaboration's tools for RCTs and crossover studies, and the checklist for nonrandomised experimental studies provided by the Johanna Briggs Institute for non-randomised experimental studies (Table 1).

Data were summarized according to the level of evidence permitted within the study design (Table 2, supplementary material 3) and the risk of bias for each study (Table 1). Evidence for each symptom was assessed following the GRADE practice recommendations [15]. If the authors disagreed on data, an external opinion was sought.

Regarding the predictably high heterogeneity of studies, no meta-analysis has been planned. 
Table 1 Assessment of the studies' risk of bias according to their designs

\begin{tabular}{|c|c|c|c|c|c|c|}
\hline$\underset{\perp}{\stackrel{N}{\perp}}$ & $\underset{+}{\stackrel{n}{\infty}}$ & $\begin{array}{l}\stackrel{+}{\prime} \\
\frac{E}{2}\end{array}$ & $\stackrel{1}{\frac{1}{Q}}$ & مُ & 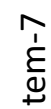 & 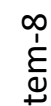 \\
\hline
\end{tabular}

Randomized controlled trials 1

NishiharaM, 2013

Cao J, 2018

Cross-over studies 2

Theobald AD, 2002

Quasi-experimental studies 3

Cankurtaran ES, 2008

Kim SW, 2008

Riechelmann RP, 2010

Ozsoy S, 2015

Ersoy MA

Davis MP, 2011

Van Gool AR, 2003

Raddin RS, 2014

Kumar N, 2017

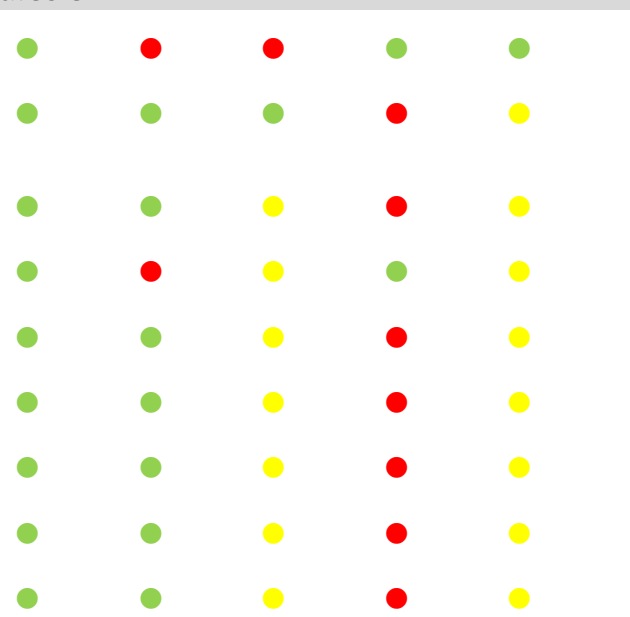

(

(

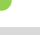

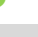

\section{$\frac{1}{E}$}

\section{(1)}


Table 2 Summary of the main findings

GRADE's quality of evidence Symptom Data summary

Targeted symptoms Low

Pain

Depression

Anxiety

Sleep disorders

Anorexia

Loss of weight

Not applicable

Breathlessness

Side effects Very low

Not applicable

Dizziness

Constipation
6 studies (236 patients) of which 1 RCT (95 patients)

Could mitigate chemotherapy-induced emesis within 3 days of treatment in addition to other anti-emetic drugs. Has not been proven to mitigate radiotherapy induced emesis. No evidence available in other situations.

4 studies (140 patients), of which 1 RCT (25 patients)

Was more effective to treat neuropathic pain from day 14 than pregabalin alone.

8 studies (249 patients)

Could be effective earlier than with compared antidepressants.

6 studies (214 patients)

Could improve anxiety, could be effective from day 15 .

5 studies (155 patients)

Could improve every stage of sleep, and extend the length of sleep. Could be efficient from week 1 .

3 studies (113 patients)

Weak evidence in effectiveness of improving appetite.

4 studies (148 patients)

Weak evidence in the effectiveness of weight gain.

1 study (17 patients)

Studies are underpowered to make a statement.

Drowsiness and fatigue 2 studies (35 patients).

The studies did not report any changes in drowsiness and fatigue, however, these two are often reported as side effects.

No study available; however, dizziness is often reported as a side effect.

No study available in this specific population, but a well-known side effect in the general population. reported was poor, with important concerns about the risk of bias (Table 2).

\section{Effectiveness of mirtazapine in cancer-related symptoms}

\section{Evidence from randomised-controlled trials}

Two studies used randomised-controlled designs, although mirtazapine was not compared with a placebo in either. The studies assessed the effectiveness of mirtazapine on two different symptoms: emesis [18] and pain [17].

Cao et al.'s study aimed to assess the effectiveness of mirtazapine in addition to usual anti-emetic therapies in the treatment of chemotherapy-induced emesis [18]. The study included 95 breast cancer patients undergoing cisplatin chemotherapy. The intervention group received mirtazapine in addition to aprepitan, a 5HT3 receptor antagonist and dexamethasone $7.5 \mathrm{mg}$. The control group received the same medications except mirtazapine. Response was assessed as "complete response to vomiting" (no emesis and no rescue treatments) and "complete control" (defined as no emesis, no rescue treatment and no more than grade 1 nausea). In the first cycle, delayed and overall complete response rates were significantly higher with mirtazapine (78.3 versus $49 \% p=$ 0.003 , and 58.7 versus $34.7 \% p=0.019)$. Similar results were observed in the 3rd cycle. The study closed early due to slow enrolment, and the interpretation of results is limited by a small sample size.

Nishihara et al.'s study compared antidepressant drugs used as adjuvants with pregabalin and opioids for intractable painful bone metastases in mixed cancer types [17]. The authors compared pregabalin $50 \mathrm{mg}$ three times daily, pregabalin $25 \mathrm{mg}$ three times daily combined with mirtazapine $7.5 \mathrm{mg}$ twice daily and pregabalin $25 \mathrm{mg}$ three times daily combined with imipramine $5 \mathrm{mg}$ twice daily. Authors also recorded the average use of opioids in the three arms. The trial included 25 patients treated for 15 days; a numerical rating scale was used to evaluate average intensity of pain and intensity of paroxysmal pain over the past $24 \mathrm{~h}$. The results found a clinically important difference over $2[26,27]$ in the total pain score intensity and in the paroxysmal pain intensity from the 1st day of use in all 3 arms of the trial. This decrease was significantly higher in the arm with mirtazapine and imipramine than in the arm with pregabalin only, and results were higher in the mirtazapine arm than the 
Fig. 1 Flow-chart (based on the CONSORT statement)

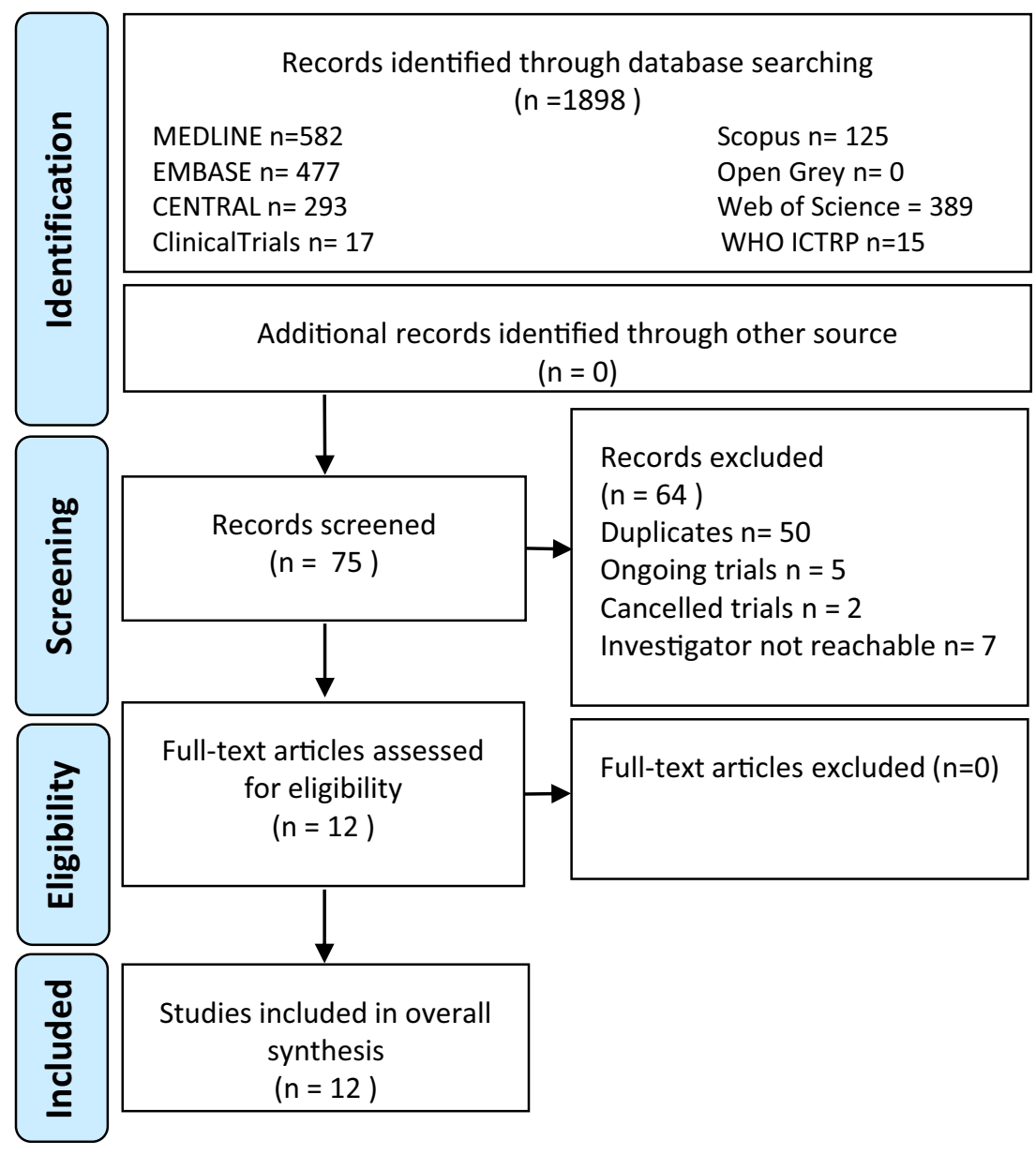

imipramine arm during the first day of treatment. There was no difference in the daily opioid dose for any of the arms.

\section{Evidence from non-randomised controlled trials}

Three non-randomised controlled trials were identified [16, $23,24]$. Two compared mirtazapine with other antidepressant drugs, one with imipramine and a control group [16], and the second with citalopram [24]. The last one compared mirtazapine with a non-interventional control group. The studies attempted to assess a wide range of symptoms including depression, anxiety, pain, appetite, emesis, insomnia, weight loss and fatigue using validated tools. Overall, the sample sizes were small (43.7 participants on average) with a high risk of bias (Table 1).

Cankurtaran et al. report a randomised-controlled trial; however, in this study, the control group was participants who had refused the intervention (mirtazapine) [16]. We have therefore considered this to be a non-randomised controlled trial. The study included 53 patients over a 6-week period with a follow-up completion rate of 0.66. Participants were cancer patients with various diagnoses. One arm received an unspecified dose of mirtazapine in addition to supportive therapy for 6 weeks, the second one received imipramine in addition to supportive therapy and the third (who had refused antidepressant treatment) received only supportive therapy. The evaluated outcomes were nausea, vomiting, reduced appetite, weight, sleep disorders, depression, anxiety and pain. Results did not show any difference in nausea or vomiting (using a single symptom scale). When assessing for pain, no difference was found between arms using a numerical rating scale. Anxiety and depression were assessed using a validated tool in cancer, the hospital anxiety and depression scale (HADS) [28, 29]. The study found a statistically and clinically significant difference [30] in anxiety $(-3.7$ points, $p=0.025)$ and depression $(-4.7$ points, $p=0.003)$ for patients taking mirtazapine, compared with imipramine and control. The effectiveness on sleep disorders was assessed using the Hamilton depression rating scale (HAM-D) which is validated in cancer [31]. For initial, middle and late insomnia, only the mirtazapine group showed improvement ( $p=0.001, p=0.001, p=0.003)$. Using single symptom scales, no significant difference was found for appetite or 
weight change in the mirtazapine group when compared with the other arms.

Raddin et al. report the results of a non-randomised controlled study including 18 patients over a 9-week period [24]. The follow-up completion rate was 0.86 . Participants received mirtazapine (starting dose $7.5 \mathrm{mg}$, escalated to 15 and then $30 \mathrm{mg}$ as appropriate) or citalopram (starting dose $10 \mathrm{mg}$, escalated to 20 and then $40 \mathrm{mg}$ as appropriate). Allocation was not concealed and was decided by clinical experience. The authors assessed depression using the Patient Health Questionnaire 9 (PHQ-9) at baseline, weeks 1, 2, 3, 4, 6 and 9. In this study, depression did not significantly improve in the overall cancer sample when evaluated using the PHQ-9. However, a sub-analysis which excluded actively dying patients showed a significant and clinically important difference [32] of $7.6(95 \% \mathrm{CI}=$ [2.9-12.2]) after 9 weeks of treatment. The quality of sleep was evaluated using the Pittsburgh Sleep Quality Index (PSQI) [33]. The study reports a non-significant improvement in sleep quality when assessed using the PSQI score $(11.0$ versus $8.6,95 \% \mathrm{CI}=[-2.2-6.9])$ and a nonsignificant improvement of the hours of sleep (5.9 versus $7.5,95 \% \mathrm{CI}=[-0.3-3.5])$. The study did not find any significant difference for weight or fatigue across the different arms.

Oszoy et al. report an open-labelled study assessing the outcomes of radiotherapy-induced cachexia treated with mirtazapine 15-30 mg for 6 months in patients with head and neck cancer [23]. The interventional group was made of patients diagnosed with major depression using the Hamilton depression rating scale, and they were compared with a control group who did not have a diagnosis of cancer or depression. The primary outcome of this study was to assess the effectiveness of mirtazapine on the level of two hormones involved in the regulation of food intake (ghrelin and leptin); secondary outcomes were assessment of weight and body mass index. The results are challenging to analyse, and no conclusion can be reached as baseline characteristics highly differ between the groups.

\section{Evidence from non-randomised non-controlled trials}

We recorded seven non-randomised non-controlled trials which were all before and after designs [19-22, 25, 34, 35]. They assessed a number of symptoms including the following: depression, anxiety, emesis, insomnia, anorexia, weight loss, breathlessness, fatigue and pain. The studies had small sample sizes (on average 24.1 participants in each study) and a high risk of bias.

Theobald et al. report a 6-week open-label crossover trial comparing the effectiveness of mirtazapine $15 \mathrm{mg}$ versus mirtazapine $30 \mathrm{mg}$ in cancer patients experiencing pain [19]. Evaluated outcomes were pain, depression, nausea and appetite. The study included 20 patients over a 6 -week period with a low follow-up completion rate (0.55). The authors assessed pain, appetite, and nausea and vomiting using a numeric rating scale and found no change for any symptom between baseline and end-point. However, patients did report feeling less concerned about their weight at week $4(\mathrm{~F}=12.9, p<0.01)$ and week $7(\mathrm{~F}=4.7, p<0.05)$ when compared with baseline. Depression was assessed using the Zung self-rating depression scale (ZSDS) which is validated for cancer [36]. The authors report a significant improvement in the ZSDS scores at week $7(\mathrm{~F}=8.2, p<0.05)$.

Ersoy et al. report a before-after trial which followed up 19 patients treated with mirtazapine $15 \mathrm{mg}$ daily for 6 months [20]. The study reports a clinically significant improvement in depression using the 17-item Hamilton rating scale with a drop from $21.4 \pm 4.9$ at baseline to $6.5 \pm 3.2$ at end-point $(p<0.001)$ [37, 38]. This improvement was significant for each sub-index of the scale rating anxiety, depression and the quality of sleep.

Riechelmann et al. report a before-after trial which followed up 21 participants for 8 weeks of treatment with mirtazapine $15-30 \mathrm{mg}$ daily [21]. The primary outcome was a gain of at least $1 \mathrm{~kg}$ after 4 weeks of treatments and secondary outcomes were appetite and quality of life. At week 4 , on intention to treat, $24 \%$ of participants had gained 1 or more kilogrammes with a median gain of $1.5 \mathrm{~kg}$ (ranging from 1 to 3.6); all respondents reported an improvement in their appetite (of more than 2 points) on the Edmonton Symptom Assessment Scale.

Kim et al. describe the results from a before-after trial which followed up 39 participants treated with mirtazapine $15 \mathrm{mg}$ daily for 4 weeks [22]. The primary outcomes were the Chonnam National University Hospital Leeds Sleep Evaluation Questionnaire (C-LESQ) for the quality of sleep, and the Clinical Global Impression (CGI) scale for nausea. The amount of sleep increased from 3.6 at baseline to $6.8 \mathrm{~h}$ per day at end-point $(p<0.001)$, the ease of getting sleep improved from 4.2 to $2.4(p<0.001)$, the quality of sleep improved from 4.3 to $2.6(p<0.001)$ and the ease of waking in the morning improved from 3.2 to $2.5(p<0.001)$. In the sub-population of patients experiencing nausea at baseline $(n=28)$, the rating of nausea decreased from $4.6 \pm 1.3$ at baseline to $2.6 \pm 1.9$ at the end-point $(p<0.001)$.

Kumar et al. present the descriptive results for a beforeafter trial including 30 patients treated with mirtazapine $7.5 \mathrm{mg}$ daily for 15 days [34]. Anorexia was a secondary outcome reported using a single symptom scale. At baseline $10.3 \%$ of participants experienced mild anorexia, $41.4 \%$ moderate anorexia and $62.1 \%$ severe anorexia. At end-point, $23.3 \%$ did not experienced anorexia anymore, $62.1 \%$ experienced mild anorexia, $13.8 \%$ moderate anorexia, and none experienced severe anorexia.

Mellar et al. report a before-after trial including 57 patients treated with mirtazapine $15 \mathrm{mg}$ daily (increased to $30 \mathrm{mg}$ daily after 1 week) for 15 days. They assessed insomnia, nausea and 
anxiety using the EORTC QLQ-C30 sub-scales and considered a response if the difference was over 1 point on the subscale. In intention to treat, insomnia and anxiety had a response rate of $33 \%$.

\section{Safety of mirtazapine's use in cancer populations}

Only two studies included a validated tool to evaluate side effects or the toxicity of mirtazapine in their design [22, 35]. Additionally, one study reported outcomes about fatigue and drowsiness using a validated scale [21] and one about the clinical global impression [25].

One open-labelled study including 42 participants with a follow-up completion rate of 0.4 used the UKU side effect rating scale [22] which has been developed to assess and rate the side effects of psychotropic treatments [39]. It has not however been validated in cancer. In this study, authors report that sleepiness/sedation was experienced after introduction in $36 \%$ of subjects. However, sleepiness/sedation appeared to decrease over the time, $19 \%$ of patients experienced increased sedation after the seven first days of treatment but they were only $8 \%$ after 14 days and none continued to experience an increased sedation on day 28 . When compared with baseline; at day $7,19 \%$ had a worst sleepiness/sedation, they were and $8 \%$ on day 14 and $0 \%$ on day 28 .

Additionally, $48 \%$ of patients already had sleepiness/ sedation before the medication. Sixty percent of those patients improved sleepiness when compared with baseline.

A non-randomised experimental study used the Common Terminology Criteria for Adverse Events to report adverse effects during the study period [35]. This tool has been developed to assess the side effects of treatments in cancer populations [40]. In this study, the author reports 4 patients experiencing grade 3 toxicity in the first week, 4 with a grade 3 toxicity in the second week and 1 with a grade 4 toxicity in the second week.

An open-labelled study including 17 participants evaluated fatigue and drowsiness using the ESAS subscales [21]. Whilst it did not find any difference in drowsiness, the ESAS fatigue subscale had a median decrease of 3.5 points, corresponding to a clinically important difference.

Overall, among all patients receiving mirtazapine and for whom the studies report the number of side effects $(n=192)$ [19-22, 24, 35], the most frequent side-effect was the somnolence/drowsiness experienced by $48 \%$ of patients $(n=$ $25)$. This concurs with the comments made in several studies reporting that sedation was the most important side-effect, responsible for the largest amount of withdrawals [18, 25]. The second most frequent side effect was dizziness which occurred in $13.4 \%(n=7)$ of the participants. The next was fatigue, experienced by $9.6 \%(n=5)$ of the patients, which was also supported by several comments found in the studies $[17,24]$. After these symptoms, by order of frequency, patients reported delirium and xerostomia, weight gain, nausea, intentional tremor, restless legs, insomnia and blurred vision. Among all studies, the withdrawals were mostly reported to be unrelated to adverse events.

Overall, only a few patients treated with mirtazapine had side effects important enough to withdraw from a study. The most frequent side effects were somnolence/drowsiness, dizziness and fatigue.

\section{Discussion}

The studies presented in this review provide low level evidence for treating polysymptomatology, limited by sample size with a high risk of bias. It is therefore not possible to recommend the use of mirtazapine for multiple palliation. However, the results confirm the effectiveness of mirtazapine in psychiatric symptoms like depression and anxiety. They are also encouraging for its effectiveness in several other symptoms, in particular, the treatment of sleep disorders, pain and cancer-related emesis. These findings should inform future RCTs to better determine the effectiveness of mirtazapine in these symptoms.

Moreover, European populations are ageing and the problem of polypharmacy is now a main concern of geriatricians [41, 42]. With an ageing population, and cancer incidence increasing with age, we can expect a rise in the number of advanced cancer patients and palliative patients undergoing polypharmacy treatments. This represents a potential risk for safety as well as for the quality of life of these patients. A key to improving the management of ageing cancer populations would be to evaluate medications that decrease the risks related to polypharmacy whilst simultaneously improving quality of life and multiple symptoms [43]. Therefore, future RCTs should aim to determine the effectiveness of alleviating multiple symptoms and quality of life.

Whilst this review did not aim to assess the effectiveness of mirtazapine in improving quality of life, four studies evaluated this as a secondary outcome, and overall, they suggested an improvement in quality of life for patients taking mirtazapine [19, 21, 24, 35]. In addition, Van Gool et al.'s paper found an increase in the clinical global impression scale, which measures the perceived efficacy of the medication in improving the global clinical state of the patient. This improvement is suggestive of a treatment response and improvement in symptom severity. Global clinical improvement might also reflect an improvement in quality of life. It supports the importance of assessing the potential improvement in quality of life whilst using this medication to alleviate multiple symptoms.

Our findings suggest that mirtazapine could be of interest in alleviating symptoms strongly associated with depressive disorders, such as anxiety and sleep disorders [44]. The population of cancer patients is at high risk of psychiatric and sleep disorders $[45,46]$, and the use of mirtazapine to alleviate 
more than one symptom could be a good alternative to multiple medications. However, effectiveness in treating these three symptoms might be explained through their categorisation as part of the same cluster of symptoms [47]. Therefore, experiencing one of these symptoms can have a worsening impact on the others [48]. For this reason, the effectiveness of mirtazapine in treating anxiety and sleep disorders could be an indirect consequence of a direct action on depressive disorders.

Regarding pain management, Nishihara's study results is informative for future research [17]. Despite a high risk of bias, the significant changes might reflect a benefit from mirtazapine in treating neuropathic pain. This effect on neuropathic pain could be of great interest in the cancer population. This population often experiences neuropathic pain, either because of a direct effect of the neoplasm or side effects of the treatments [49]. Moreover, chronic pain and especially chronic neuropathic pain are common risk factors for depressive disorders [50], and some authors suggest that, considering that they are part of the same symptom cluster, an improvement in neuropathic pain may lead to an improvement in sleep quality [51]. Therefore, the effectiveness of mirtazapine in chronic neuropathic pain management could be of interest in more ways than one by treating the underlying symptom cluster of pain-depression-sleep disorders. Serotonin noradrenaline reuptake inhibitors (SNRIs) are antidepressants approved to treat neuropathic pain. Their action on neuropathic pain is not fully understood; however, it might be mediated by enhancing serotonin and noradrenaline in the spinal and supraspinal structures [52]. Besides, tricyclic antidepressants are also approved in this indication. Like mirtazapine, tricyclic antidepressants inhibit serotonin and noradrenaline reuptake in the synapse, resulting in a central noradrenergic and serotoninergic neurotransmission increase [53]. These shared pharmacological pathways between mirtazapine and other medications licenced for treating neuropathic pain could explain the potential effectiveness of mirtazapine for this indication.

Mirtazapine may also be an interesting antidepressant to treat multiple symptoms because of its effects on appetite and weight [14]. Mirtazapine's side effects might be of great interest, particularly because malnutrition is a cause of treatment intolerance and shortens the life expectancy of advanced cancer patients [54]. For these reasons, mirtazapine may be the preferred option when treating depression in cancer patients. To date, evidence for the use of mirtazapine to improve weight gain and appetite is lacking but studies are currently ongoing to address this.

Another interesting symptom for which no treatment is licensed in Europe is breathlessness. Evidence is lacking to support the use of mirtazapine in alleviating breathlessness; however, some pilot studies have shown encouraging results in alleviating breathlessness in advanced lung disease conditions, including lung cancers [55]. Mirtazapine appears to be a promising candidate to pursue, but definitive randomized controlled trials are required to determine its efficacy and safety in this setting.

\section{Limitations}

Our review has several limitations. Whilst including grey literature, we cannot be certain that we have identified all studies. Some studies were excluded from the review because the data was not available. Publication bias is a common concern in interventional studies, especially in populations with life-threatening diseases, as many studies do not recruit or retain enough patients to have strong results, limiting their publication in peerreviewed journals. Therefore, this review may have been impacted by publication bias. Additionally, we excluded studies that did not focus only on cancer patients. This decision was supported by the fact that most of these studies had "cancer-affected patients" as exclusion criteria. However, this choice potentially led to the neglect of relevant data.

\section{Conclusion}

Overall, there are limited studies which aim to assess the effectiveness of mirtazapine in alleviating multiple symptoms in the cancer population and no studies which assess the use of mirtazapine to treat polysymptomatology. The study designs are mostly too weak to support strong results and often only include a small sample size. However, these results should inform further large RCTs which are able to determine the effectiveness of mirtazapine in treating multiple symptoms in the cancer population.

Authors' contributions IJH had the original idea, supervised the design of the work, the acquisition and interpretation of the data and revised the article.

GE designed the protocol, acquired the data, interpreted the data and drafted the article.

$\mathrm{NL}$ acquired the data, interpreted the data and revised the article.

$\mathrm{AJ}$ interpreted the data and revised the article.

Funding information This systematic review has been funded by King's College London, the employer of the investigators.

Availability of data and material The datasets analysed during the current study are available from the corresponding author on reasonable request.

\section{Compliance with ethical standards}

Competing interests The authors declare that they have no competing interests. 
Open Access This article is licensed under a Creative Commons Attribution 4.0 International License, which permits use, sharing, adaptation, distribution and reproduction in any medium or format, as long as you give appropriate credit to the original author(s) and the source, provide a link to the Creative Commons licence, and indicate if changes were made. The images or other third party material in this article are included in the article's Creative Commons licence, unless indicated otherwise in a credit line to the material. If material is not included in the article's Creative Commons licence and your intended use is not permitted by statutory regulation or exceeds the permitted use, you will need to obtain permission directly from the copyright holder. To view a copy of this licence, visit http://creativecommons.org/licenses/by/4.0/.

\section{References}

1. Bausewein C, Booth S, Gysels M, Kühnbach R, Haberland B, Higginson IJ (2010) Understanding breathlessness: cross-sectional comparison of symptom burden and palliative care needs in chronic obstructive pulmonary disease and cancer. J Palliat Med 13:11091118. https://doi.org/10.1089/jpm.2010.0068

2. Kierner KA, Weixler D, Masel EK, Gartner V, Watzke HH (2016) Polypharmacy in the terminal stage of cancer. Support Care Cancer 24:2067-2074. https://doi.org/10.1007/s00520-015-3007-z

3. Teunissen SCCM, Wesker W, Kruitwagen C, de Haes HC, Voest EE, de Graeff A (2007) Symptom prevalence in patients with incurable cancer: a systematic review. J Pain Symptom Manag 34: 94-104. https://doi.org/10.1016/j.jpainsymman.2006.10.015

4. Currow DC, Abernethy AP, Allcroft P, Banzett RB, Bausewein C, Booth S, Carrieri-Kohlman V, Davidson P, Disler R, Donesky D, Dudgeon D, Ekstrom M, Farquhar M, Higginson I, Janssen D, Jensen D, Jolley C, Krajnik M, Laveneziana P, McDonald C, Maddocks M, Morelot-Panzini C, Moxham J, Mularski RA, Noble S, O'Donnell D, Parshall MB, Pattinson K, Phillips J, Ross J, Schwartzstein RM, Similowski T, Simon ST, Smith T, Wells A, Yates P, Yorke J, Johnson MJ (2016) The need to research refractory breathlessness. Eur Respir J 47:342-343. https://doi.org/10.1183/ 13993003.00653-2015

5. Davis MP, Khawam E, Pozuelo L, Lagman R (2002) Management of symptons associated with advanced cancer: olanzapine and mirtazapine. Expert Rev Anticancer Ther 2:365-376. https://doi. org/10.1586/14737140.2.4.365

6. Rougé Bugat M-E, Bourgouin M, Gérard S, Lozano S, Brechemier D, Cestac P, Cool C, Balardy L (2017) Drug prescription including interactions with anticancer treatments in the elderly: a global approach. J Nutr Health Aging 21:849-854. https://doi.org/10.1007/ s12603-017-0946-8

7. Nightingale G, Skonecki E, Boparai MK (2017) The impact of polypharmacy on patient outcomes in older adults with cancer. Cancer J 23:211-218. https://doi.org/10.1097/PPO. 0000000000000277

8. Lovell N, Ward J (2011) The role of mirtazapine in treating multiple symptoms in patients with cancer. Clin Focus Cancer Med 2:61-64

9. Kent JM (2000) SNaRIs, NaSSAs, and NaRIs: new agents for the treatment of depression. Lancet 355:911-918. https://doi.org/10. 1016/S0140-6736(99)11381-3

10. Anttila SA, Leinonen EV (2001) A review of the pharmacological and clinical profile of mirtazapine. CNS Drug Rev 7:249-264

11. Lovell N, Wilcock A, Bajwah S, Etkind SN, Jolley CJ, Maddocks M, Higginson IJ (2019) Mirtazapine for chronic breathlessness? A review of mechanistic insights and therapeutic potential. Expert Rev Respir Med 13:173-180. https://doi.org/10.1080/17476348. 2019.1563486
12. Zaini S, Guan NC, Sulaiman AH, Zainal NZ, Huri HZ, Shamsudin SH (2018) The use of antidepressants for physical and psychological symptoms in cancer. Curr Drug Targets 19:1431-1455. https:// doi.org/10.2174/1389450119666180226125026

13. Carvalho AF, Sharma MS, Brunoni AR, Vieta E, Fava GA (2016) The safety, tolerability and risks associated with the use of newer generation antidepressant drugs: a critical review of the literature. Psychother Psychosom 85:270-288. https://doi.org/10.1159/ 000447034

14. Biswas PN, Wilton LV, Shakir SAW (2003) The pharmacovigilance of mirtazapine: results of a prescription event monitoring study on 13554 patients in England. J Psychopharmacol (Oxf) 17:121-126. https://doi.org/10.1177/0269881103017001716

15. Balshem H, Helfand M, Schunemann HJ et al (2011) GRADE guidelines: 3. Rating the quality of evidence. J Clin Epidemiol 64: 401-406. https://doi.org/10.1016/j.jclinepi.2010.07.015

16. Cankurtaran ES, Ozalp E, Soygur H, Akbiyik DI, Turhan L, Alkis N (2008) Mirtazapine improves sleep and lowers anxiety and depression in cancer patients: superiority over imipramine. Support Care Cancer 16:1291-1298. https://doi.org/10.1007/s00520-0080425-1

17. Nishihara M, Arai Y-CP, Yamamoto Y et al (2013) Combinations of low-dose antidepressants and low-dose pregabalin as useful adjuvants to opioids for intractable, painful bone metastases. Pain Phys 16:E547-E552

18. Cao J, Wang B, Wang $Z$ et al (2018) Efficacy of mirtazapine in preventing delayed nausea and vomiting induced by highly emetogenic chemotherapy: an open-label, randomized, multicenter phase III trial. J Clin Oncol 36:1078-1078. https://doi.org/10.1200/ JCO.2018.36.15_suppl.1078

19. Theobald DE, Kirsh KL, Holtsclaw E, Donaghy K, Passik SD (2002) An open-label, crossover trial of mirtazapine (15 and 30 $\mathrm{mg}$ ) in cancer patients with pain and other distressing symptoms. J Pain Symptom Manag 23:442-447

20. Ersoy MA, Noyan AM, Elbi H (2008) An open-label long-term naturalistic study of mirtazapine treatment for depression in cancer patients. Clin Drug Investig 28:113-120. https://doi.org/10.2165/ 00044011-200828020-00005

21. Riechelmann RP, Burman D, Tannock IF, Rodin G, Zimmermann C (2010) Phase II trial of mirtazapine for cancer-related cachexia and anorexia. Am J Hosp Palliat Med 27:106-110. https://doi.org/10. 1177/1049909109345685

22. Kim S-W, Shin I-S, Kim J-M, Kim YC, Kim KS, Kim KM, Yang SJ, Yoon JS (2008) Effectiveness of mirtazapine for nausea and insomnia in cancer patients with depression. Psychiatry Clin Neurosci 62:75-83. https://doi.org/10.1111/j.1440-1819.2007. 01778.x

23. Ozsoy S, Besirli A, Unal D, Abdulrezzak U, Orhan O (2015) The association between depression, weight loss and leptin/ghrelin levels in male patients with head and neck cancer undergoing radiotherapy. Gen Hosp Psychiatry 37:31-35. https://doi.org/10. 1016/j.genhosppsych.2014.09.002

24. Raddin RS, Park EM, Hamer RM, Nelson KM, Mayer DK, Rosenstein DL, Bernard SA (2014) A pilot study to evaluate symptom-oriented selection of antidepressants in patients with cancer. J Palliat Med 17:167-175. https://doi.org/10.1089/jpm.2013. 0412

25. Van Gool AR, Bannink M, Stronks DL, Vos MS (2003) Re: Mirtazapine in cancer patients. J Pain Symptom Manag 25:7-8

26. Farrar JT, Young JP, LaMoreaux L et al (2001) Clinical importance of changes in chronic pain intensity measured on an 11-point numerical pain rating scale. Pain 94:149-158. https://doi.org/10.1016/ S0304-3959(01)00349-9

27. Farrar JT, Portenoy RK, Berlin JA et al (2000) Defining the clinically important difference in pain outcome measures. Pain 88:287294. https://doi.org/10.1016/S0304-3959(00)00339-0 
28. Hartung TJ, Friedrich M, Johansen C, Wittchen HU, Faller H, Koch U, Brähler E, Härter M, Keller M, Schulz H, Wegscheider K, Weis J, Mehnert A (2017) The hospital anxiety and depression scale (HADS) and the 9-item patient health questionnaire (PHQ-9) as screening instruments for depression in patients with cancer: depression screening in oncology. Cancer 123:4236-4243. https:// doi.org/10.1002/cner.30846

29. Vodermaier A, Millman RD (2011) Accuracy of the hospital anxiety and depression scale as a screening tool in cancer patients: a systematic review and meta-analysis. Support Care Cancer 19: 1899-1908. https://doi.org/10.1007/s00520-011-1251-4

30. Puhan MA, Frey M, Büchi S, Schünemann HJ (2008) The minimal important difference of the hospital anxiety and depression scale in patients with chronic obstructive pulmonary disease. Health Qual Life Outcomes 6:46. https://doi.org/10.1186/1477-7525-6-46

31. Olden M, Rosenfeld B, Pessin H, Breitbart W (2009) Measuring depression at the end of life: is the Hamilton depression rating scale a valid instrument? Assessment 16:43-54. https://doi.org/10.1177/ 1073191108320415

32. Kroenke K, Wu J, Yu Z, Bair MJ, Kean J, Stump T, Monahan PO (2016) Patient health questionnaire anxiety and depression scale: initial validation in three clinical trials. Psychosom Med 78:716727. https://doi.org/10.1097/PSY.0000000000000322

33. Lu T, Li Y, Pan J, Wu D (2013) Study on minimal important difference of the Pittsburgh sleep quality index based on clinical trial of traditional Chinese medicine. Chin J Integr Med

34. Kumar N, Barai S, Gambhir S, Rastogi N (2017) Effect of mirtazapine on gastric emptying in patients with cancerassociated anorexia. Indian J Palliat Care 23:335-337. https://doi. org/10.4103/IJPC.IJPC_17_17

35. Davis MP, Kirkova J, Lagman R, Walsh D, Karafa M (2011) Intolerance to mirtazapine in advanced cancer. J Pain Symptom Manag 42:e4-e7. https://doi.org/10.1016/j.jpainsymman.2011.05. 002

36. Dugan W, McDonald MV, Passik SD, Rosenfeld BD, Theobald D, Edgerton S (1998) Use of the Zung self-rating depression scale in cancer patients: feasibility as a screening tool. Psychooncology 7: 483-493. https://doi.org/10.1002/(SICI)1099-1611(199811/12)7: 6<483::AID-PON326>3.0.CO;2-M

37. Zimmerman M, Posternak MA, Chelminski I (2005) Is the cutoff to define remission on the Hamilton rating scale for depression too high? J Nerv Ment Dis 193:170-175

38. Montgomery SA, Möller H-J (2009) Is the significant superiority of escitalopram compared with other antidepressants clinically relevant? Int Clin Psychopharmacol 24:111-118. https://doi.org/10. 1097/YIC.0b013e32832a8eb2

39. Lingjærde O, Ahlfors UG, Bech P et al (1987) The UKU side effect rating scale: a new comprehensive rating scale for psychotropic drugs and a cross-sectional study of side effects in neuroleptictreated patients. Acta Psychiatr Scand 76:1-100. https://doi.org/ 10.1111/j.1600-0447.1987.tb10566.x

40. Trotti A, Colevas A, Setser A, Rusch V, Jaques D, Budach V, Langer C, Murphy B, Cumberlin R, Coleman CN, Rubin P (2003) CTCAE v3.0: development of a comprehensive grading system for the adverse effects of cancer treatment. Semin Radiat
Oncol 13:176-181. https://doi.org/10.1016/S1053-4296(03) 00031-6

41. Hajjar ER, Cafiero AC, Hanlon JT (2007) Polypharmacy in elderly patients. Am J Geriatr Pharmacother 5:345-351. https://doi.org/10. 1016/j.amjopharm.2007.12.002

42. Wehling M (2011) Guideline-driven polypharmacy in elderly, multimorbid patients is basically flawed: there are almost no guidelines for these patients: letters to the editor. J Am Geriatr Soc 59: 376-377. https://doi.org/10.1111/j.1532-5415.2011.03252.x

43. Rosini M (2014) Polypharmacology: the rise of multitarget drugs over combination therapies. Future Med Chem 6:485-487. https:// doi.org/10.4155/fmc. 14.25

44. Redeker NS, Lev EL, Ruggiero J (2000) Insomnia, fatigue, anxiety, depression, and quality of life of cancer patients undergoing chemotherapy. Sch Inq Nurs Pract 14:275-290 discussion 291-298

45. Brintzenhofe-Szoc KM, Levin TT, Li Y, Kissane DW, Zabora JR (2009) Mixed anxiety/depression symptoms in a large cancer cohort: prevalence by cancer type. Psychosomatics 50:383-391. https://doi.org/10.1176/appi.psy.50.4.383

46. Otte JL, Carpenter JS, Manchanda S, Rand KL, Skaar TC, Weaver M, Chernyak Y, Zhong X, Igega C, Landis C (2015) Systematic review of sleep disorders in cancer patients: can the prevalence of sleep disorders be ascertained? Cancer Med 4:183-200. https://doi. org/10.1002/cam4.356

47. Fan G, Filipczak L, Chow E (2007) Symptom clusters in cancer patients: a review of the literature. Curr Oncol Tor Ont 14:173-179

48. Theobald DE (2004) Cancer pain, fatigue, distress, and insomnia in cancer patients. Clin Cornerstone 6:S15-S21. https://doi.org/10. 1016/S1098-3597(05)80003-1

49. Fallon MT (2013) Neuropathic pain in cancer. Br J Anaesth 111: 105-111. https://doi.org/10.1093/bja/aet208

50. Torta R, Ieraci V, Zizzi F (2017) A review of the emotional aspects of neuropathic pain: from comorbidity to co-pathogenesis. Pain Ther 6:11-17. https://doi.org/10.1007/s40122-017-0088-z

51. Enomoto T, Yamashita A, Torigoe K, Horiuchi H, Hirayama S, Nakahara K, Yanase M, Sakai H, Ikegami D, Nagase H, Suzuki T, Iseki M, Inada E, Narita M (2012) Effects of mirtazapine on sleep disturbance under neuropathic pain-like state. Synapse 66:483-488. https://doi.org/10.1002/syn.21532

52. Marks D, Shah M, Patkar A, Masand PS, Park GY, Pae CU (2009) Serotonin-norepinephrine reuptake inhibitors for pain control: premise and promise. Curr Neuropharmacol 7:331-336. https://doi. org/10.2174/157015909790031201

53. Obata $H$ (2017) Analgesic mechanisms of antidepressants for neuropathic pain. Int J Mol Sci 18:2483. https://doi.org/10.3390/ ijms 18112483

54. Argilés JM (2005) Cancer-associated malnutrition. Eur J Oncol Nurs 9:S39-S50. https://doi.org/10.1016/j.ejon.2005.09.006

55. ISRCTN - ISRCTN32236160: Better treatments for refractory breathlessness. http://www.isrctn.com/ISRCTN32236160. Accessed 25 Apr 2019

Publisher's note Springer Nature remains neutral with regard to jurisdictional claims in published maps and institutional affiliations. 